\title{
Toward a Data Scalable Solution for Facilitating Discovery of Science Resources
}

\author{
Jesse Weaver ${ }^{\mathrm{a}, *}$, Vito Giovanni Castellana ${ }^{\mathrm{a}}$, Alessandro Morari ${ }^{\mathrm{b}}$, Antonino \\ Tumeo $^{\mathrm{a}}$, Sumit Purohit ${ }^{\mathrm{a}}$, Alan Chappell ${ }^{\mathrm{b}}$, David Haglin ${ }^{\mathrm{a}}$, Oreste Villa ${ }^{\mathrm{c}}$, \\ Sutanay Choudhury ${ }^{a}$, Karen Schuchardt ${ }^{a}$, John Feo ${ }^{a}$ \\ ${ }^{a}\{j e s s e . w e a v e r$, vitoGiovanni.castellana, antonino.tumeo, \\ sumit.purohit, david.haglin, sutanay.choudhury, \\ karen.schuchardt, john.feo\}@pnnl.gov \\ Pacific Northwest National Laboratory \\ Richland, WA 99354, USA \\ ${ }^{b}\{$ alessandro.morari, alan.chappell $\} @ p n n l . g o v$ \\ Pacific Northwest National Laboratory \\ Seattle, WA 98109, USA \\ ${ }^{c}$ ovilla@nvidia.com \\ NVIDIA Research \\ Santa Clara, CA 95051, USA
}

\begin{abstract}
Data-intensive science simultaneously derives from and creates the need for large quantities of data. As such, scientists increasingly need to discover and analyze new datasets from diverse sources. Beyond the sheer volume of data, issues posed by the resultant data heterogeneity are often overlooked. We postulate that heterogeneity challenges can be solved (at least in part) with the adoption of the Resource Description Framework (RDF), a graphbased data model. In turn, this requires scalable graph query systems for discovering and analyzing data. Consequently, we investigate GEMS, a graph engine for large-scale clusters. We describe the features of GEMS that make it suitable for answering graph queries and scaling to larger quantities of data. We evaluate GEMS' ability to answer real science-based queries over real-world, curated, science metadata. We also demonstrate GEMS' ability to scale to larger datasets using a benchmark.
\end{abstract}

Keywords: data intensive, science metadata, graph database, scalability,

*Corresponding author: jesse.weaver@pnnl.gov

Preprint submitted to Parallel Computing

August 15, 2014

(C) 2014. This manuscript version is made available under the Elsevier user license http://www.elsevier.com/open-access/userlicense/1.0/ 
semantics

\section{Introduction}

Data-intensive science simultaneously derives from and creates the need for large quantities of data [1]. As such, scientists increasingly need to discover and analyze new datasets from diverse sources. Beyond the sheer volume of data, issues posed by the resultant data heterogeneity are often overlooked. Examples include: vocabularies in Comma-Separated Values (CSV) [2] and Resource Description Framework (RDF) [3] formats from the Global Change Master Directory (GCMD) [4]; NetCDF [5] data files from the Atmospheric Radiation Measurement (ARM) [6] climate research facility; and web pages from the International Soil Moisture Network (ISMN) [7].

Data heterogeneity challenges have been addressed (at least in part) in the Semantic Web (or Web of Data) field through various recommendations including the Resource Description Framework (RDF) [3]. RDF has been adopted in some scientific communities $[8,9,10,11,12]$, including in our own previous work [13]. While it helps with the heterogeneity challenges, the adoption of RDF exacerbates the volume challenges. A recent benchmark study [14] suggests that the largest, surmountable RDF datasets for most modern RDF databases are 1-10 billion RDF triples (graph edges) in size, and at those quantities, performance is significantly degraded. In our own work, a curation of science metadata has generated 1.4 billion triples, yet that constitutes only a small sample of the available science metadata. In order to handle even greater quantities of RDF triples with less cost to performance, many RDF databases (and relevant research) are moving toward parallel, cluster-based systems $[15,16,17,18,19,20,21,22,23]$. A similar trend is also occuring with generic graph databases [24, 25].

Consequently, we are investigating a distributed, graph-based query system being developed at Pacific Northwest National Laboratory (PNNL) called the Graph Engine for Multithreaded Systems (GEMS) [26]. Postulating that the RDF data model is sufficient for solving the heterogeneity problems, we seek to evaluate how well GEMS can handle the problem of ever-growing volumes of data (i.e., data scaling [27]). GEMS stores RDF triples in distributed, in-memory indexes in such a way as to quickly and naturally support parallel graph walking, the fundamental operation for graph queries. It is built atop our custom Global Memory and Threading (GMT) [28] runtime system for clusters, designed from the ground up to tolerate the distributed, random 
data accesses that naturally occur when walking a distributed graph. GEMS is a work in progress, but our results herein demonstrate GEMS' capability and potential for scaling queries to large graphs.

As an extension of [13], the novel contributions of this work are as follows.

- An extended and more detailed description of the real-world, dataintensive, science-based use case first introduced in [13].

- An updated description of the GEMS software stack previously introduced in $[13,29]$. Specifically, our graph data structure has changed radically, and we no longer use distributed hashmaps for storing results.

- A more in-depth evaluation using GEMS to answer queries on science metadata than we were previously able to provide in [13], also using a dataset that is over ten times larger.

- An evaluation of GEMS' scalability using generated benchmark datasets. Unlike in [26], this paper evaluates on individual query times rather than (parallel) query throughput.

- A performance comparison between GEMS and Urika [30].

The rest of the paper is organized as follows. In Section 2, we describe an example of curating science metadata into the RDF data model so as to provide background for the kind of data over which we wish to query. We briefly introduce the RDF data model, the SPARQL query language [31], give a concrete example of the curated metadata, and provide example queries. Section 3 describes GEMS at a high level with emphasis on the characteristics of GEMS that improve data scalability of graph walking, the fundamental operation for answering graph-based queries over the curated science metadata introduced in Section 2. Section 4 presents experimental results for: (1) the performance of GEMS to answer queries over science metadata; (2) the scalability of GEMS to handle larger datasets using a benchmark; and (3) a performance comparison with Urika [30]. Section 5 covers related works, and conclusions and future work are discussed in Section 6.

\section{Curated Science Metadata from RDESC}

The investigation herein is motivated by ever-growing, heterogeneous, science metadata. In this section, we describe efforts in curating (i.e., coping 
with the heterogeneity of) science metadata as part of the Resource Discovery for Extreme Scale Collaboration (RDESC) project. We include this description for two reasons: (1) to establish and give an example of data heterogeneity in science; and (2) to provide background for the RDESC metadata that will be used to evaluate GEMS in Section 4.2.

\subsection{RDF for metadata}

Facilitating discovery of science resources requires semantically meaningful integration of otherwise disparate, heterogeneous metadata. These metadata are available in different binary or syntactic formats (e.g., variables and attributes in NetCDF [5] files, attributes in HDF [32] files, "hidden" web services, embedded in HTML pages, or even simple text) and usually do not have clear semantic interoperability (e.g., measurements for the same property can implicitly have different units, or variations in definitions of altitude). In order to integrate the metadata, we first establish a precise understanding of the metadata and then attempt to capture those semantics in an ontology (see Figure 1). To integrate the metadata, we convert it from its native form to RDF triples - a graph-based data model that is flexible enough for nearly (if not truly) any kind of metadata.

As the term implies, RDF triples consist of three (ordered) parts: subject, predicate, and object. This simple data model constitutes a labeled, directed multigraph. A triple S P 0 uniquely identifies a directed edge in the graph going from the vertex uniquely identified by $\mathrm{S}$ to the vertex uniquely identified by $0 . P$ is effectively the edge label.

At present, the RDESC metadata consists of nearly 1.4 billion RDF triples, mostly metadata about ARM [6] data streams and GeoNames [33] locations, although it includes to a lesser proportion descriptions of Global Change Master Directory (GCMD) [4] locations, keywords, and datasets, as well as metadata from the International Soil Moisture Network (ISMN) [7]. Specifically, the RDESC metadata contains descriptions of 781,750 data resources, which can be broadly broken down into descriptions of 777,230 datasets (the vast majority from ARM), 2,767 catalogs (all from ARM), 1,701 data centers (mainly from GCMD and ARM), and 52 data networks (most from ISMN).

An example of the RDF triples from ARM is depicted in Figure 2. $\mathrm{fkb30ecorM1.b1} \mathrm{identifies} \mathrm{a} \mathrm{datastream} \mathrm{in} \mathrm{ARM.} \mathrm{DataStream} \mathrm{is} \mathrm{a} \mathrm{subclass}$ of Catalog which is a subclass of DataResource. The ARM datastream is 
located at the $\mathrm{fkb} . \mathrm{M} 1$ facility in Black Forest, Germany, and its data is derived from the $\mathrm{fkb} 30$ smplcmask1zwangM1 instrument which is a cloud mask from micropulse lidar. It has measured properties (locally) named $f c, h$, and lv_e which have associated time ranges and classifications. All three measured properties are categorized as pertaining to "surface flux", but whereas $f c$ measures the "rate of flow for carbon dioxide", $h$ and lv_e measure "the time rate of flow for the specific enthalpy difference between two phases of a substance at the same temperature." These metadata were originally available in an accumulation of NetCDF [5] files, each file being semantically isolated.

While this example provides the gist of the kind of metadata, more complex features are not illustrated herein. For example, we include in our metadata the GeoNames [33] metadata in order to make use of its spatial subsumption hierarchy. We also have an ARM-specific portion of the ontology that extends the high-level ontology shown in Figure 1. Instances of measured properties are also connected to broad categories - like "soil moisture" - in order to enable users to ask broad queries - like "find all data resources having measurements related to soil moisture." Through explicitly labeled connections, we can understand the relationships between entities and use those labels to pose semantically meaningful questions, which is the topic of the following section.

\section{2. $S P A R Q L$ for queries}

Once metadata is in RDF form, we can query it using SPARQL [31], the recommended RDF query language. In this section, we briefly introduce SPARQL primarily for the purpose of expressing our queries in a standard, graph-oriented way.

The fundamental query operation in SPARQL is matching basic graph patterns (BGPs), which is similar to constrained subgraph isomorphism. A BGP is a set of triple patterns, and a triple pattern is like a RDF triple in which any term can instead be a variable (indicated by starting with a ?). SPARQL also supports property paths. Property paths are a way of describing paths in a graph based on edge labels. A property path containing P1/P2 indicates successive path traversals where first path P1 must be walked, and then from the destination node of P1, a P2 path must be walked. A property path $\mathrm{P} *$ indicates that zero or more $\mathrm{P}$ paths be traversed. A property path $\mathrm{P}$ ? indicates that walking a $\mathrm{P}$ path is optional, and a property path (P1|P2) 


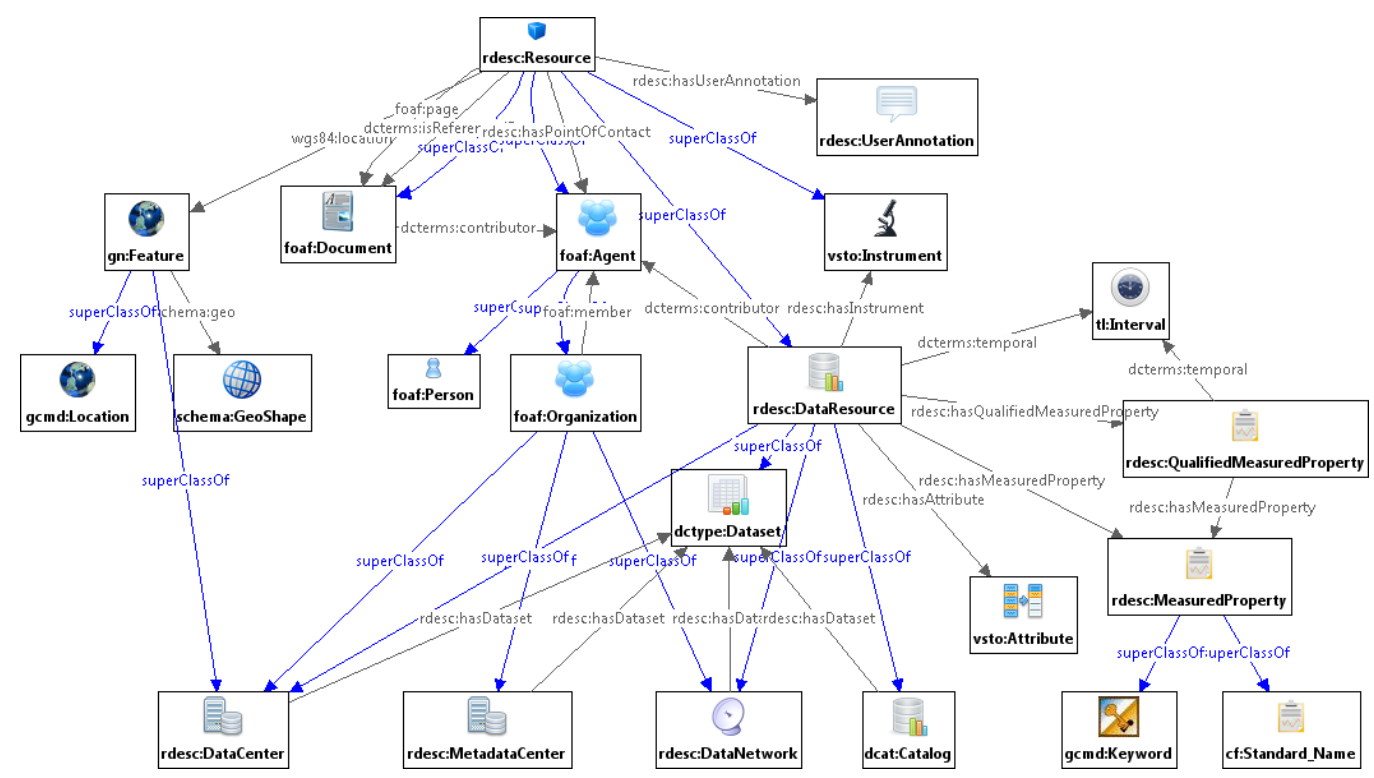

Figure 1: The high-level RDESC ontology

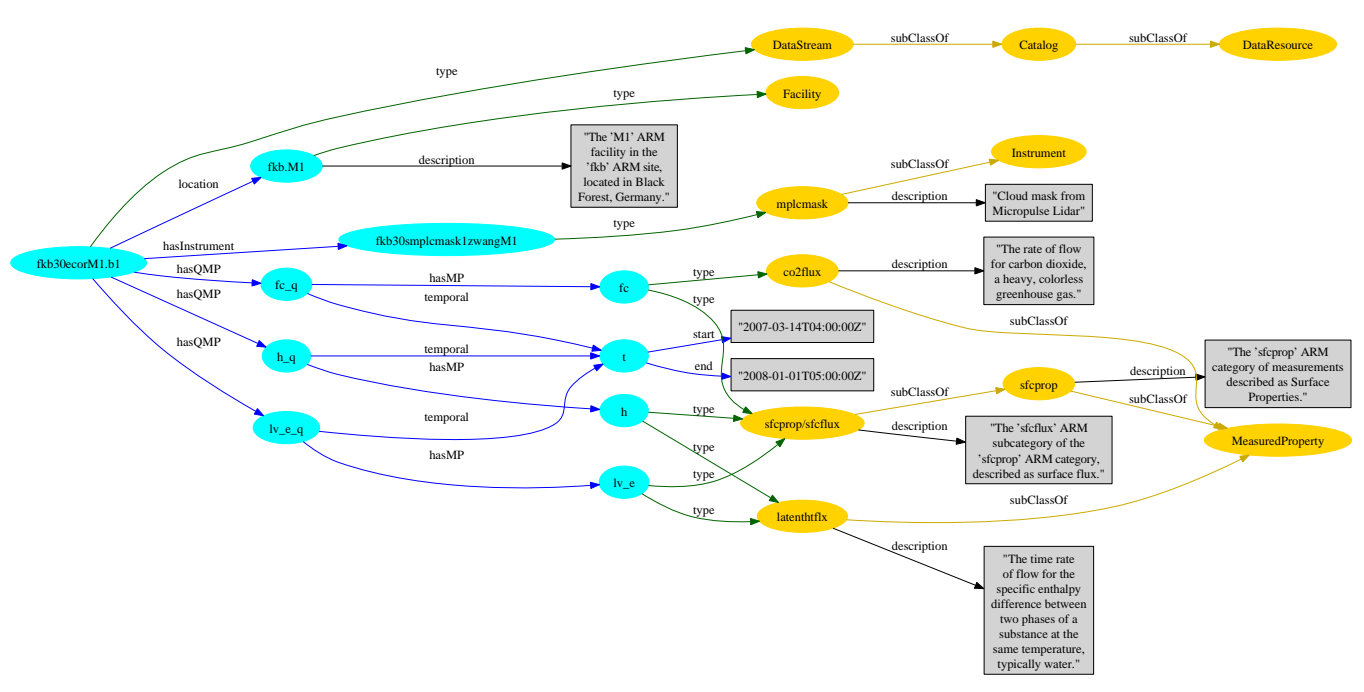

Figure 2: Example ARM metadata curated into a RDF graph in RDESC 
indicates that either a P1 or a P2 path must be walked. A property path is ultimately grounded in edge labels (predicate terms).

An (syntactically simplified) example of a RDESC query is given in Figure 3 and visually depicted in Figure 4, which asks for all instruments related to data resources containing measurements related to soil moisture. The triple pattern on line 2 says to match all paths in the graph where the destination vertex is DataResource, which is preceded (in the path) by zero or more edges labeled subClassOf, preceded by one edge labeled type, the source vertex of which can be any vertex since ?RES is a variable. ?RES is now bound to vertexes that identify data resources. From the ?RES vertex, on line 3, we then traverse one outgoing edge labeled has Instrument to find the instrument associated with the data resource. Line 4 walks from the data resource vertexes to theird measured properties, to their classification (or "type"), and walks up the class hierarchy to determine whether the measured properties are related to soil moisture. For those data resources that are related to soil moisture, on line 5, we look up the locations at which the measurements were taken by walking from the data resource vertexes across an outgoing edge labeled either location or locatedIn, and then zero or more edges labeled either parentFeature or locatedIn. Line 5 climbs the spatial subsumption heirarchy of GeoNames so that the data resource will be associated at various levels of regions (e.g., city, state, country, continent). ${ }^{1}$

A more natural question for a domain scientist to ask is given in Figure 5. This query is essentially the same as the first one with the added SPARQL features GROUP BY, HAVING, and COUNT. GROUP BY ?LOC says to group the results together by their locations, and HAVING (COUNT (DISTINCT ?INST) >= 10) says to choose only those locations that have at least 10 different instruments. The SELECT part defines the output of the query which in this case is a two-tuple ?LOC ?POINTS where ?POINTS is defined as COUNT (DISTINCT ?INST), i.e. the number of distinct instruments per location.

\section{GEMS}

Having established data heterogeneity in science by way of example in Section 2, and having illustrated that the graph-based RDF data model can

\footnotetext{
${ }^{1}$ Note that this description of property paths is non-compliant with the current recommendation of SPARQL 1.1 [31], but instead conforms to an earlier working draft of SPARQL 1.1 [34].
} 


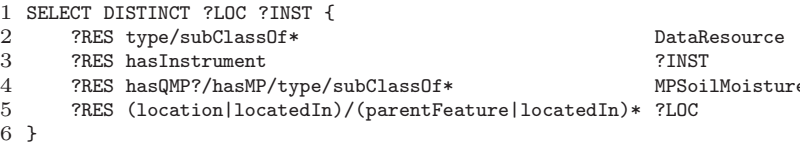

Figure 3: Find all instruments related to data resources containing measurements related to soil moisture

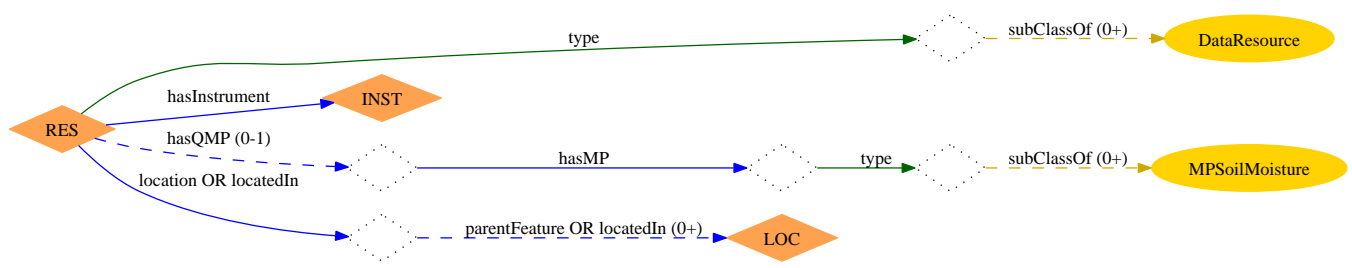

Figure 4: Visual depiction of the graph portion of the query

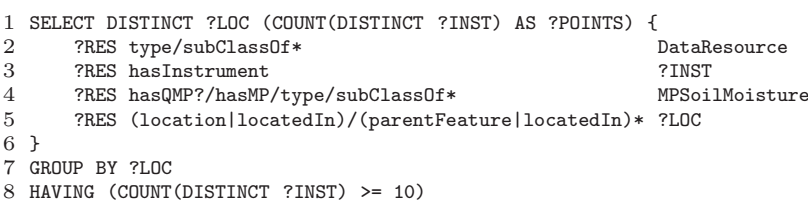

Figure 5: Find all locations having measurements related to soil moisture that are taken with at least ten instruments

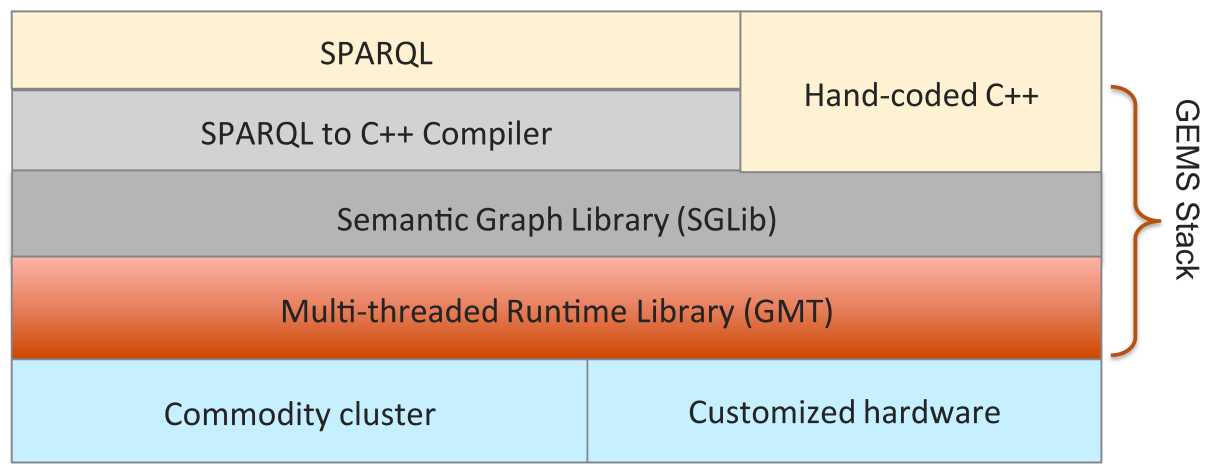

Figure 6: The architecture of our software system for semantic graph databases 
help, we proceed in this section to describe GEMS with emphasis on the features that make it suitable for scaling graph queries to larger datasets. GEMS is a multiple layer stack (Figure 6). Its three layers are: (1) a SPARQL-toC++ compiler; (2) a multithreaded, semantic graph library (SGLIB); and (3) a custom multithreaded runtime (GMT) for large-scale clusters. The following sections describe each layer.

\subsection{SPARQL-to-C++ compiler}

The front end of our architecture translates SPARQL queries into $\mathrm{C}++$ code that utilizes SGLIB, as well as the runtime methods of GMT. Processing queries on the RDF graph is achieved using a constrained subgraph isomorphism algorithm expressed as a collection of nested first-order function calls, one for each edge in the pattern. An example of such a function is given in Figure 7, which is an example of the translation of ?RES type/subClassOf* DataResource from Figures 3 and 5. Note that we start edge traversals from the DataResource vertex and walk "backwards" across incoming edges instead of starting at any vertex and walking "forwards" across outgoing edges. Starting from a known vertex allows us to constrain the search space. The execute function is the entry point into the query.

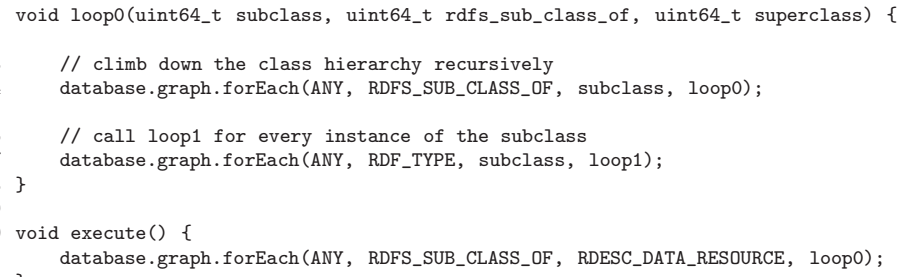

Figure 7: Example portion of $\mathrm{C}++$ code generated by the compiler

The forEach method is a parallel construct that creates a task per matching edge, and each task is scheduled for parallel execution by GMT. Note the recursive call to loop0 in the first forEach on line 4 of Figure 7. Such recursive calls implement property path queries in a manner that enables us to easily support complex, graph-oriented features. However, this naive approach is limited to cases where cycles do not occur when matching the property path. We are currently adding support for handling cycles.

Our compiler is not yet complete but we are already finding many opportunities for optimization. We have still to perform the type of query planning 
done by modern database systems, and optimization of SPARQL algebra expressions as defined in the W3C standard [31] and in recent research like [35]. While we make use of statistics about the graph when translating SPARQL to $\mathrm{C}++$ by hand, we are just now incorporating such information in our compiler to decide where to begin the graph walk, to select the order of traversal (which loop to call next), and to predict the size of intermediate and final results. As an example of how we use statistics to support heuristic optimizations, given a graph pattern with two triple patterns, if only one matches with a supporting index (see the next section for details), it is best to start the walk with that one. If both are supported by indexes and we know how many matches there are for each, we should start with the triple pattern having the fewest matches in order to constrain the number of matches for the second one (assuming they share a variable). These basic heuristics are meant to reduce the overall number of edge traversals.

\section{2. $S G L I B$}

The data structures implemented within the SGLIB layer support query answering using the features provided by the GMT layer. When large datasets are loaded, the size of the SGLIB data structures are expected to be larger than what can fit into the memory of a single node. Therefore, we implement the SGLIB data structures (mostly) using the global memory of GMT. The fundamental data structures are graph, dictionary, and table.

The ingest phase of the GEMS workflow initializes a graph and dictionary. The dictionary is used to associate string labels with unique integer identifiers (UIDs). Therefore, each RDF triple is encoded as a sequence of three UIDs. The graph indexes RDF triples in subject-predicate-object and objectpredicate-subject orders. Each index is range-partitioned so that each node in the cluster gets an almost equal number of triples. Subject-predicate and object-predicate pairs with highly skewed proportions of associated triples are automatically specially distributed among nodes so as to avoid load imbalance as a result of data skew. At present, we use a simple heuristic for testing skewedness of subject-predicate (resp. object-predicate) pairs. Given the average count and the standard deviation of the count for such pairs (which we compute automatically before building the indexes), we simply say that a pair that occurs more often than threshold = average + stddev times is highly skewed (although the threshold is configurable). For those pairs, we range partition the associated triples across all nodes. In effect, there is a range partitioning for each highly skewed subject-predicate (resp. 
object-predicate) pair, and then there is another range partition over all the other triples that are not associated with a highly skewed pair.

As previously described in Section 3.1, the bulk of the query is executed as a parallel graph walk. The forEach method is used to call a function for all matching edges/triples, in parallel. Thus, the graph walk can be conceptualized as nested loops. The nested loop structure can result in the exponential generation of parallel tasks that are well-suited to the runtime features implemented in GMT for tolerating latencies across global memory. At the end (or "bottom") of a graph walk, results are buffered in a loader object that is associated with a table object. When all the parallel edge traversals complete, the loader finalizes by actually inserting the results into its associated table. At this point, operations like deduplication (DISTINCT) and grouping (GROUP BY) are performed using the table. Results are effectively structs containing primitive members for storing values of variables. Many variables will be bound to UIDs, but some may contain primitive values.

Internally, the forEach method has to make two decisions: (1) whether new tasks should be spawned; and (2) if tasks should be spawned, on which nodes they should be spawned. It is sometimes the case that tasks will be spawned on the nodes where relevant portions of the graph reside, but in some cases, tasks are spawned randomly across cluster nodes in order to improve load balancing. The process for making such determinations is as follows. First, the range partitioning table (which is replicated on all nodes) is accessed to determine which nodes in the cluster could have matching triples. If it happens that every node could have matching triples (as in the case of triple patterns matching highly skewed subject-predicate or objectpredicate pairs), then a task is spawned to every node in the cluster to iterate (usually in parallel, by generating more local tasks) to call back to the user-supplied function for each matching triple. Otherwise, each node with possibly matching triples is interrogated with a remote function call to determine whether any matching triples actually exist. The remote function calls are made in parallel, and the calling task waits until all the results are received. The result of this function call can vary between four cases.

- Stop. The return value indicates that the remote node actually has no matching triples.

- Result. The remote node had exactly one matching triple, and the match is returned in the return value. In this case, the calling task will call back to the user-provided function with the matching triple. 
- Location. The remote node returns the location of matching triples in the global memory of GMT. In this case, the calling task will generate new tasks, one for each match. The new tasks are spawned randomly across the cluster in order to avoid load imbalance, and they use global memory access methods to fetch a matching triple and call back to the user-provided function.

- Inconveyable. If matching triples cannot be accessed in global memory (usually because part of the match exists in indexes that are not in the global memory space), then the remote node returns a message indicating that it has matching triples but that it can provide no other information. In this case, the calling task will generate a new task at the remote node which will interrogate the local indexes and call back the user-provided function with matching triples.

Stop and result are the best cases because the cost is a single remote function call. In the case of location, the remote function call must be followed by spawning tasks. In the case of inconveyable, the remote function call must be followed by at least two spawns: the first to create a task that interrogates the indexes of the remote node, which will then spawn more tasks for iterating over matching triples in parallel. Inconveyable is the worst case; it is very expensive due to limitations on our current implementation, which we are trying to resolve. However, the inconveyable case only happens when a triple pattern is presented for which there is no appropriate index (e.g., subject ANY predicate) or there are two or more ANYs. We rarely encounter such cases in query execution, but we do include such a case in the evaluation later in this paper (BSBM Q3).

\section{3. $G M T$}

GMT is a custom runtime system at the base of our GEMS stack that provides a global address space and lightweight software multithreading [28]. These features - in combination with network message aggregation - make GMT well-designed for parallel, distributed graph walking. Starting from a graph vertex (or set of vertexes), the traversal of each incident edge can occur in parallel, and thus many tasks can be generated. These tasks may perform remote data accesses or function calls that cause them to block (as previously described in Section 3.2), but in that case, a thread will context switch

to a task that is ready for execution (if available). Thus communication is 


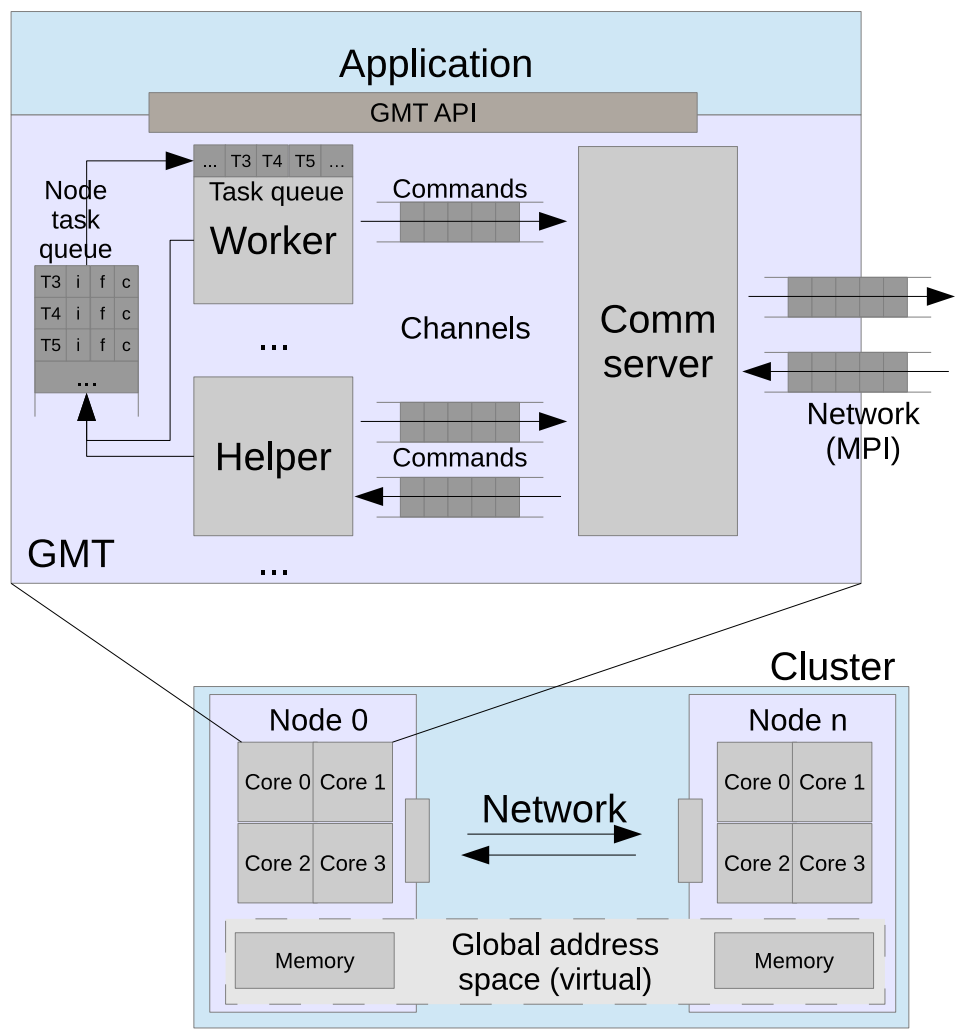

Figure 8: Overview of GMT architecture

hidden when there is enough parallel work. Although, commands for remote data accesses and function calls generate fine-grained communication operations, they get aggregated before being transmitted across the network, thus improving utilization of network bandwidth. GMT is described in greater detail in the remainder of this section.

GMT is designed to run on any multi-node, Linux-based, commodity cluster, integrating x86 processors and supporting MPI. Figure 8 shows an overview of the GMT architecture. GMT creates three different types of threads:

- Worker: executes the application code, partitioned into lightweight tasks, and generates outgoing requests.

- Helper: manages incoming requests, and generates the appropriate replies.

- Communication Server: effectively executes the data transfers through 
MPI.

Each instance hosts multiple worker and helper threads (usually in the same amounts), and a single communication server. All tasks that are generated in GMT are executed by worker threads. GMT distributes tasks across the nodes of the cluster as determined by a policy that is specified (by SGLIB, or generally, any GMT user) when the tasks are requested to be spawned. Tasks are usually generated as a block of iterations from a parallel for-loop, although in some cases, a one-off task is spawned (like with the inconveyable case from Section 3.2). The spawning of these tasks are actually serialized as commands and aggregated with other commands (other spawns and get/put data accesses) going to the same node. When the buffer for the aggregated commands fills up (or if a timeout occurs), the communication server then sends the contents of the buffer to the destination node, where it is received by that node's communication server. ${ }^{2}$ At the destination node, helper threads service the commands. If the command is merely a data access, the helper performs the operation and returns the result (or simply an acknowledgment in some cases). In the case of spawning new tasks, the helper places the summary of those tasks (i.e., the block of iterations from the parallel forloop, or the one-off task itself) in the node task queue. When workers have open slots in their local task queues, they take tasks (sometimes only some of the iterations) from the node task queue and place them in their local task queues. A worker thread executes only the tasks in its local queue. When a task is blocking (perhaps waiting for a remote data access, or waiting for a child task to complete), the worker context switches to the next "ready" task in its queue and begins executing it. In this way, communication can be overlapped with computation, and thus when there is enough parallel work to be done, communication can essentially come for free (of course, there is the overhead of context switching).

Note that tasks in the node task queue are essentially compressed since they are summarized. This has the added benefit that GEMS does not cause every edge traversal to immediately occupy additional space in memory. In database terms, this equates to delaying materialization of intermediate results until they are actually needed, thus providing the potential to save a

\footnotetext{
${ }^{2}$ GMT integrates a two-level message aggregation: a thread (worker or helper) first aggregates commands towards the same remote node; when local queues are full, GMT aggregates them for the entire node before sending them out. This increases the size of transferred messages, maximizing network bandwidth utilization.
} 
significant amount of space compared to the execution of conventional joins that often require the materialization of the input relations (which could be intermediate results).

\section{Evaluation}

In this section, we evaluate the performance of GEMS in two ways. First, in Section 4.2, to demonstrate GEMS' ability to aide discovery of science resources, we use GEMS to answer RDESC queries on the RDESC dataset. However, since the RDESC dataset is real data, it cannot be arbitrarily scaled to different sizes in order to demonstrate data scaling. Therefore, as a second part of the evaluation, in Section 4.3, we evaluate GEMS using the BSBM [36] benchmark which allows us to observe how well GEMS can scale to larger datasets by adding more nodes to the cluster. Although BSBM does not reflect a science use case (it is actually designed around a business intelligence use case), to the best of our knowledge, it is the most suitable benchmark currently available for testing scalability of SPARQL queries on RDF datasets. Using BSBM will also allow future works to compare performance. However, there are admittedly some drawbacks in using BSBM for our purposes, mainly: (1) the data does not reflect heterogeneity of multiple sources (it is built on the idea that products, reviews, etc. are structured and named in a uniform way); and (2) it does not use property paths, which were commonly needed in RDESC.

For a comparison with some of the state of the art, we compare performance of executing the BSBM queries with Urika [30] in Section 4.4. A more complete comparison with the state of the art would include comparison with other cluster-based solutions like 4store [16] and Virtuoso Cluster Edition [15] which we were unable to include for this work due to the current setup at PNNL Institutional Computing (PIC). We are currently trying to resolve those issues.

Details of the underlying hardware and relevant software for GEMS are given in Section 4.1 to help provide context for the experimental results.

\subsection{Experimental setup}

All GEMS experiments reported in this work were executed on Pacific Northwest National Laboratory's Olympus supercomputer. Olympus is a cluster of 604 nodes interconnected through a QDR Infiniband switch with 648 ports (theoretical peak of $4 \mathrm{~GB} / \mathrm{s}$ ). Each of Olympus' nodes features two 
AMD Opteron 6272 processors at $2.1 \mathrm{GHz}$ and $64 \mathrm{~GB}$ of DDR3 memory clocked at 1,600 MHz. Each socket hosts eight processor modules (two integer cores, one floating point core) on two different dies, for a total of 32 integer cores per node. A module includes a L1 instruction cache of $64 \mathrm{~KB}$, two L1 data caches of $64 \mathrm{~KB}$, and a 2 MB L2 cache. Each 4-module die hosts a shared L3 cache of $8 \mathrm{MB}$. Dies and processors communicate through HyperTransport. Olympus is configured with Red Hat 5.5 and Linux Kernel 2.6.32. The code was compiled using gcc and $\mathrm{g}++$ version 4.6 .2 with the -03 optimization flag, and GMT used OpenMPI 1.6.3. The file system is a Lustre file system $(50 \mathrm{~GB} / \mathrm{s})$, and input files were striped at 1MB across 12 Object Storage Targets (OSTs).

\subsection{Discovery of Science Resources}

In this section, we describe some results in testing the use of GEMS to answer queries for RDESC. Since the SPARQL-to-C++ compiler presently has support for only the more basic SPARQL features, we hand-coded the queries in a similar fashion as we would expect from a mature version of the compiler. The queries from Section 2 were simple examples. Figure 9 is a complete query from RDESC (in this case using the precise SPARQL syntax with implied PREFIX declarations). When hand-coded using the SGLIB API, these queries (including comments and print statements) were roughly 250 lines of code, except for the query in Figure 9 which was roughly 450 lines of code.

Referring to Table 1, using 8, 16, and 32 nodes of Olympus (equating to 120, 240, and 480 GMT workers, respectively), the query in Figure 9 correctly produces no results since there is currently no data that matches the query, in the range of 2.04-5.29 seconds (on average). Although there are no results, the query still needs to walk paths of several edges before determining there are no results.

Queries in Figures 3 and 5, and the additional exploratory query in Figure 10, instead, under the same circumstances, run (on average) in the ranges of $0.492-1.74,0.585-0.706$, and 8.16-15.9 seconds with 76, 1, and 68 results, respectively. For the query in Figure 5, the one location with at least 10 "points" (instruments) for soil moisture data is the ARM facility sgp.X1 (that is, the first experimental facility in ARM's southern great plains site). Queries from Figures 9 and 10 decrease in time as nodes are added, while queries from Figures 3 and 5 have less predictable behavior. In particular, the query from Figure 3 has a significant spike in query time on 16 nodes 


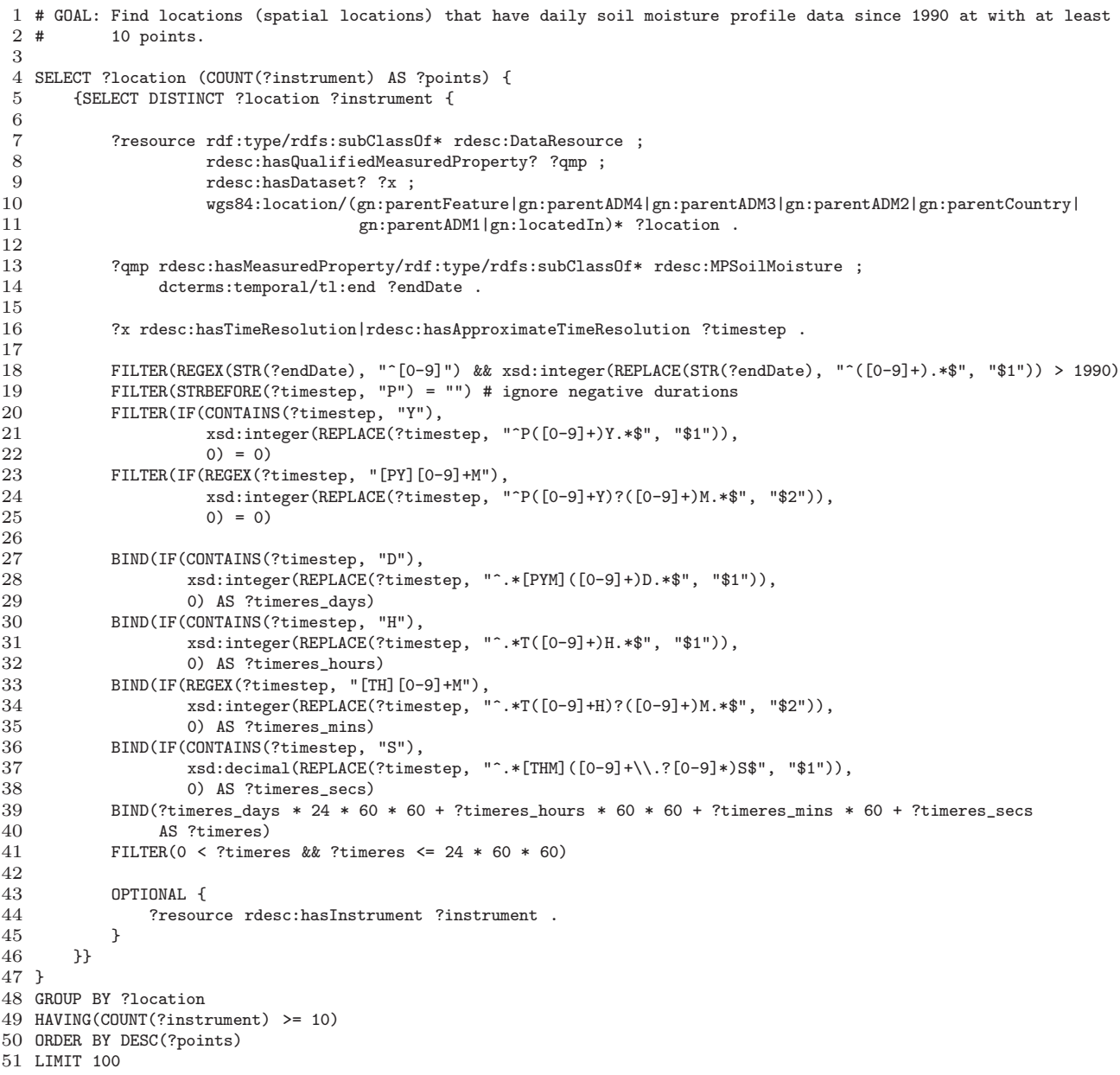

Figure 9: A complete RDESC use case query

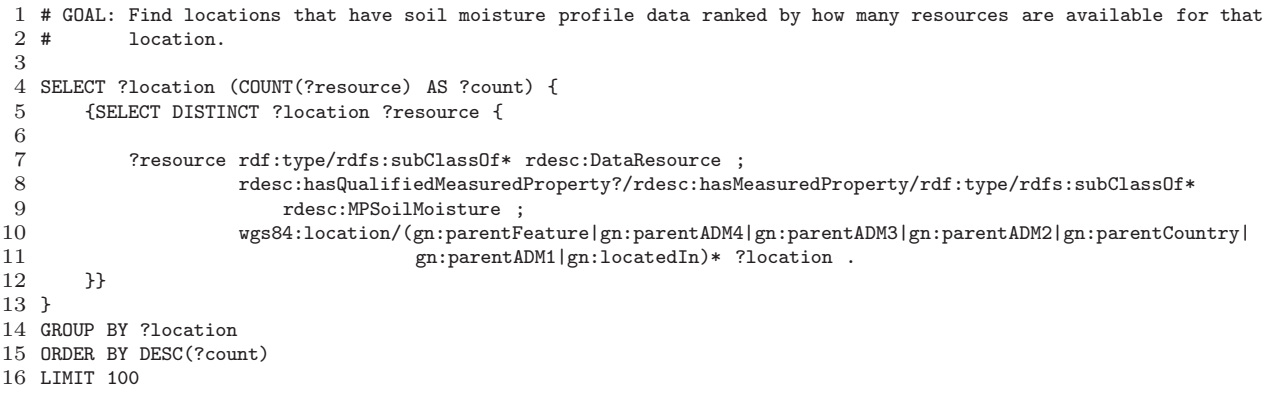

Figure 10: A complete RDESC exploratory query 


\begin{tabular}{|l|c|c|c|c|}
\hline & \#results & 8 nodes & 16 nodes & 32 nodes \\
\hline Ingest & & 1218 & 580 & 406 \\
Query Fig.3 & 76 & 0.492 & 1.74 & 0.724 \\
Query Fig.5 & 1 & 0.585 & 0.706 & 0.690 \\
Query Fig.9 & 0 & 5.29 & 4.93 & 2.04 \\
Query Fig.10 & 68 & 15.9 & 9.38 & 8.16 \\
\hline
\end{tabular}

Table 1: Average ingest and query times (in seconds) over 10 runs, on fixed RDESC dataset of nearly 1.4B triples, varying number of nodes

which we continue to investigate. Regardless, this portion of the experiment demonstrates the ability of GEMS to answer science-oriented queries over large, real-world metadata in seconds.

\subsection{Scalability}

By virtue of being real-world data, the RDESC dataset cannot arbitrarily scale in size for benchmarking purposes, so we include herein an evaluation using the Berlin SPARQL Benchmark (BSBM) [36] in order to provide a more complete analysis of the scalability of GEMS. Again, since the SPARQL-to$\mathrm{C}++$ compiler is under development, we hand-coded the queries for this evaluation. However, we hand-coded the queries in a fashion that we expect a mature version of our compiler to be able to generate.

BSBM defines a set of SPARQL queries and datasets to evaluate the performance of RDF databases. BSBM datasets are based on a business intelligence use case involving product types, producers, vendors, offers, and reviews. We run queries 1 to 6 (see Figures 11 through 16) on datasets with $100 \mathrm{M}, 1 \mathrm{~B}$, and 10B triples, on up to 128 nodes. The BSBM queries are actually query templates in which certain terms must be selected. For our instantiations of Q1 through Q6, we made sure to choose valid values for instantiating the queries. To make sure we had at least one low selectivity query (that is, a query that would have to search a large portion of the RDF graph), we instantiated BSBM Q1 with country: US which is the lowest selectivity instantiation of BSBM Q1.

Table 2 shows the time to ingest the datasets and the execution times of the queries on $100 \mathrm{M}, 1 \mathrm{~B}$, and $10 \mathrm{~B}$ triples, increasing the number of cluster nodes to accommodate more data. The ingest times are visually depicted in Figure 17c. Sizes of the input files respectively are 20.6 GB (100M), 208 GB (1B), and 2.10 TB (10B). In all cases, the ingest time decreases as the number of nodes increases. 


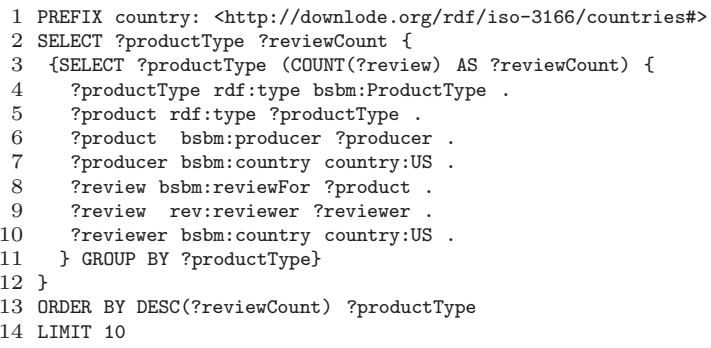

Figure 11: Instantiation of BSBM Q1: the top 10 most discussed product categories of products from the US based on number of reviews by reviewers from the $U S$

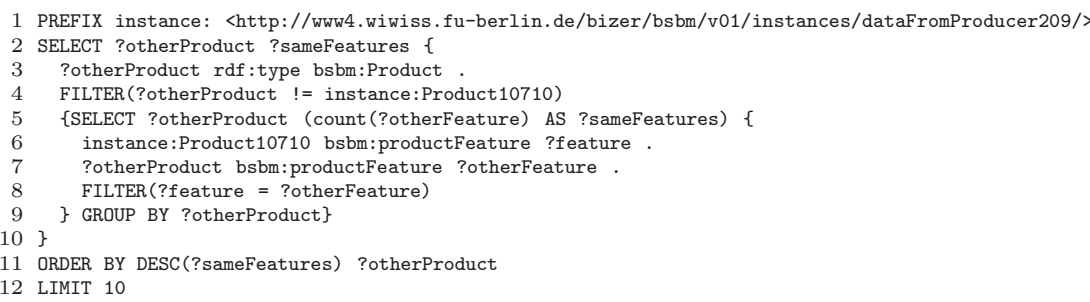

1 ORDER BY DESC(?sameFeatures) ?otherProduct

12 LIMIT 10

Figure 12: Instantiation of BSBM Q2: the top 10 products most similar to Product10'10, rated by the count of features they have in common

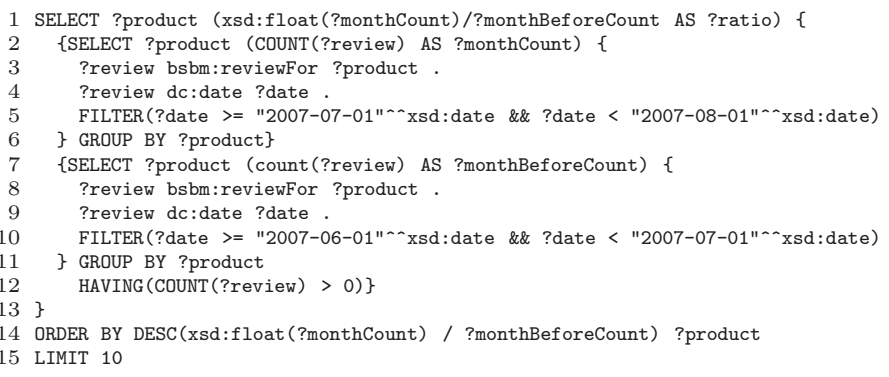

Figure 13: Instantiation of BSBM Q3: products with largest increase of interest (ratio of review counts) from June 2007 to July 2007 


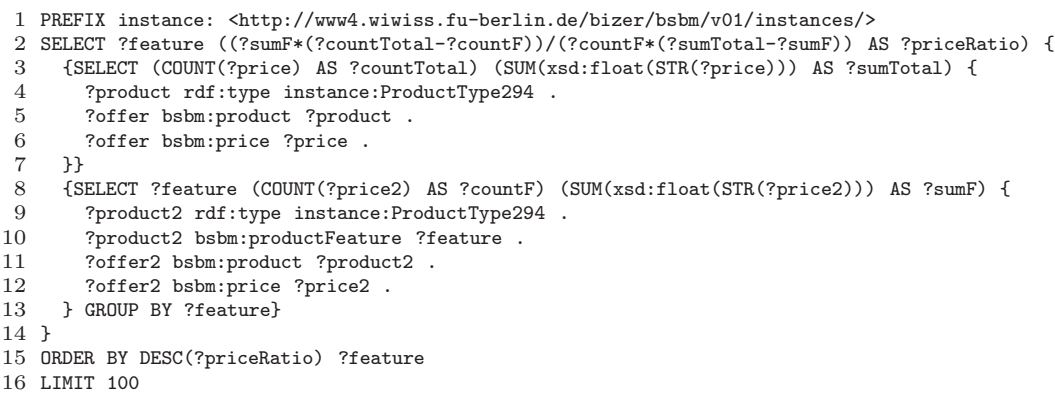

15 ORDER BY DESC(?priceRatio) ?feature

16 LIMIT 100

Figure 14: Instantiation of BSBM Q4: for ProductType294, features with the highest ratio between price with that feature and price without that feature

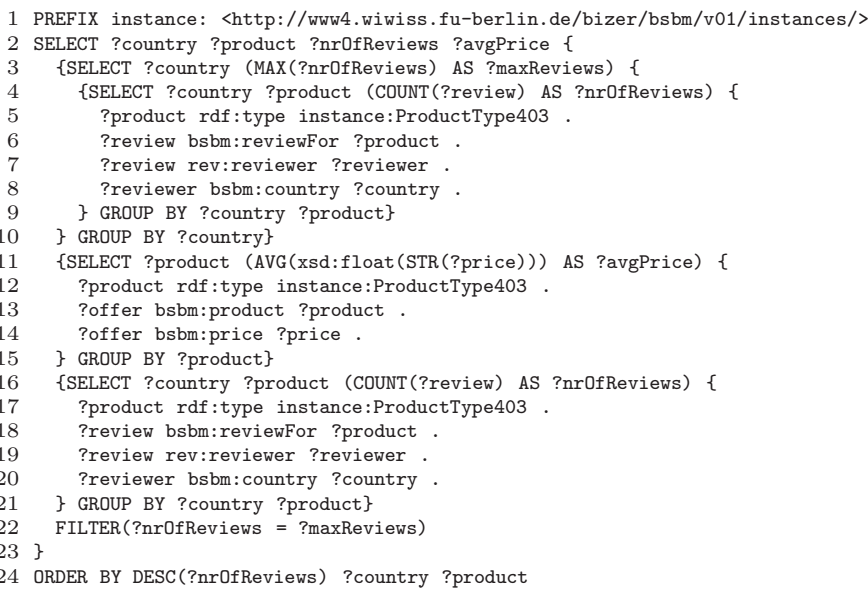

Figure 15: Instantiation of BSBM Q5: shows the most popular products of type ProductType 403 for each country, by review count 


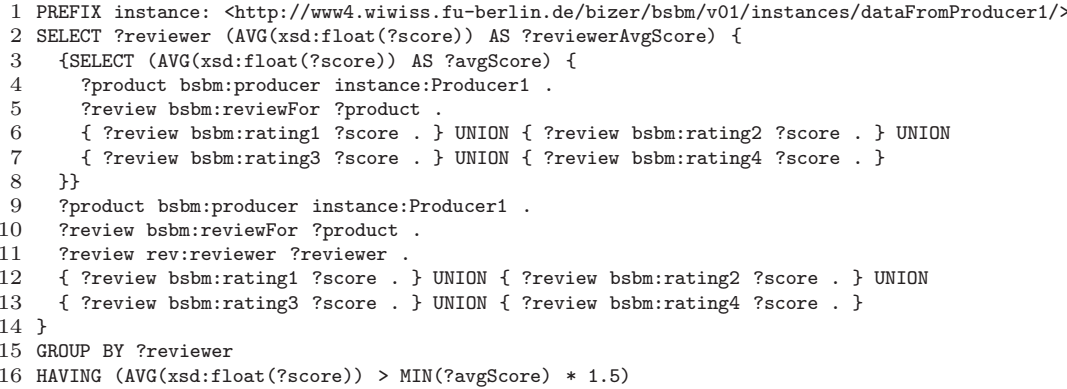

Figure 16: Instantiation of BSBM Q6: reviewers who rated products by Producer1 much higher than other reviewer

The query times for $100 \mathrm{M}$ and $1 \mathrm{~B}$ are visually depicted in Figures 17a and 17b, respectively. Q1 must search a large portion of the graph, so there is significant parallel work available. As a result, the query time for Q1 decreases as more nodes are added. Q3 also searches a large portion of the graph, but for a different reason. Because Q3 does not contain a constant vertex from which to start the graph walk, we begin by iterating (in parallel) over all the vertices in the graph. That is, we unnecessarily touch all of the graph, and for this reason, there is a lot of parallel work, and our implementation of Q3 scales as the number of nodes increases. We could effectively remove the unnecessary work by adding a predicate index to GEMS, but in practice, we have found that a predicate index is rarely necessary. Adding another index to the two that we already have (subject-predicate-object and object-predicate-subject) would increase the graph's memory footprint by roughly $50 \%$, and so far, we have not been convinced that it is a worthwhile tradeoff. Q2, Q4, and Q5 take roughly the same amount of time or take slightly longer when adding nodes. The reason is that these queries touch a relatively small portion of the graph and cannot produce enough parallelism to make use of the extra computing resources obtained by adding nodes to the cluster. Not only that, but adding nodes actually increases the average cost of remote data accesses, and as a result, the query time can increase a little. This effect is best illustrated by Q6 which touches a very small part of the graph. In fact, we can see from Figure 17d that growing the dataset does not significantly increase the time (for 16 nodes, the query from $100 \mathrm{M}$ to $1 \mathrm{~B}$ triples increased by only 0.042 seconds).

Figure 17d shows the times for the most (Q3) and least (Q6) scalable 
queries for all datasets. Q3 is clearly impacted by growing the dataset. Note that at 16 nodes, when growing the dataset by a factor of ten (from 100M to $1 \mathrm{~B}$ ), the runtimes increased only by factors of 5.64 (Q3) and 1.13 (Q6). There are two main points here. The first is that nodes can be added to the cluster in order to accommodate larger datasets. The second is that, if sufficient memory is available, the query times do not grow in direct linear proportion to the size of the data, at least for the case considered in this evaluation. This is likely due to the GMT layer being able to better overlap computation and communication in the graph search. By adding more data (and in the case of Q3, more work), the threads have more work to do while waiting for communication.

\subsection{Performance Comparison}

To compare with some of the state of the art, in this section, we provide an experimental performance comparison with Urika [30] using the BSBM queries. We did not use the RDESC queries on Urika because it does not support property paths and therefore would not be able to answer these queries.

YarcData defines Urika as an appliance for data/graph analytics. It effectively is a multi-node system (although not quite in the conventional cluster sense) for data analytics which integrates the Cray XMT 2 with a database front end based on the Jena framework. Details about the Cray XMT can be found in [37]. Like in our GMT layer, multithreading in the Cray XMT 2 provides latency tolerance when accessing remote data. The global address space allows the database layer to be implemented as if working on a shared memory system, without caring for partitioning the data or balancing the workload across nodes. Our Urika system has 128 nodes and a total memory of 4 TB. Each node has 32 GB of DDR2 memory with a data rate of 800 MHz. The Threadstorm 2 processors run at $500 \mathrm{MHz}$. Because the system exploits a shared memory abstraction, even when Urika executes on a single node, the database and the queries can still use the memory of the entire machine. The version of the full Urika stack we used for our benchmarks was 6.0.4.

We compare ingest and query execution time of GEMS to that achieved with Urika in Table 3 and Figure 18. A completely fair comparison between a conventional commodity cluster and specialized hardware like the Cray XMT 2 is essentially impossible. Since we are motivated by growing dataset sizes, we chose to compare the systems having equivalent available memory. 
Our Urika appliance has 4 TB of shared memory, and so we compared using GEMS with 64 nodes which - at 64 GB per node - provides a collective global memory of 4 TB. The Cray XMT 2 has an advantage in that it supports extremely fast context switching at the hardware level, whereas GMT supports context switching more slowly in software. However, our commodity cluster has the advantage that - whenever possible - locality can be exploited in the conventional cache hierarchies, a feature completely preempted in the design of the Cray XMT 2. These are just some of the architectural differences to consider when interpreting the results of the comparison.

We assume that the time reported by Urika includes the time to convert the SPARQL query into a plan, execute it, and then return results. Therefore, in an attempt to compare as fairly as possible, we consider two cases with GEMS. The first is the case in which a single query will be used frequently and need only be compiled once. In this case, we assume the cost of the SPARQLto- $\mathrm{C}++$ compilation (including the $\mathrm{C}++$ compilation itself) will ultimately be amortized to a negligible value, and so we only report the query time (which includes the time to decode results). This time is referred to simply as "GEMS" in Table 3 and Figure 18. The other case we considered is the case in which one-time exploratory queries are being performed, in which case we must include the compilation time. In this case, we also include the time to compile the hand-coded queries using mpic ++ , and we refer to those times as "GEMS w/mpic++" in Table 3 and Figure 18.

In the case of frequently reused queries (i.e., when ignoring compilation time), GEMS outperforms Urika for all queries. Compared to Urika, GEMS takes from $7.19 \%$ (Q6) to $72.4 \%$ (Q1) of the time that Urika does. When accounting for compilation times, GEMS takes from 29.1\% (Q2) to $123 \%$ (Q5) of the time that Urika does. In all cases (even with compilation time), GEMS is roughly on par or faster than Urika, except for Q1 in which GEMS $\mathrm{w} /$ mpic++ takes 1.80 seconds longer. Aside from query time, there are other factors that can be taken into account when comparing with Urika. Commodity clusters are likely to be more affordable (in terms of purchase and maintenance) and more available than specialized hardware. Also, to our knowledge, the Urika appliance can run only one query at a time. In previous work [26], we have shown that running queries in parallel on GEMS achieves a high query throughput. Admittedly, though, the GEMS interface is not fully mature, so at present, it is more difficult to use. 


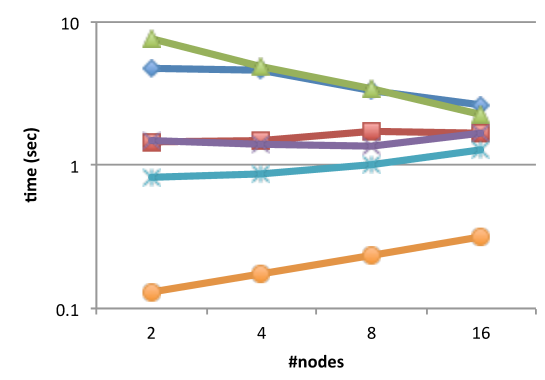

(a) Average query times, over three runs, for 100M triples on 2-16 nodes

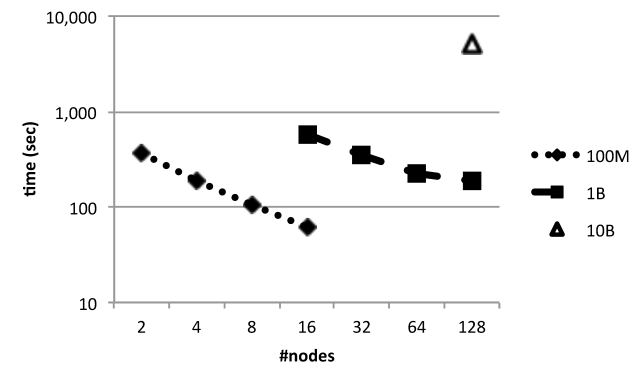

(c) Ingest times

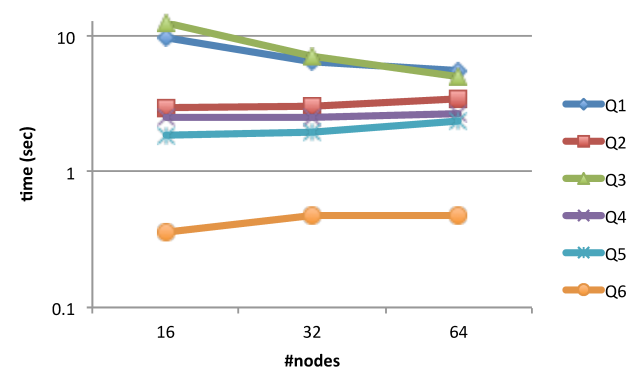

(b) Average query times, over three runs, for $1 \mathrm{~B}$ triples on 16-64 nodes

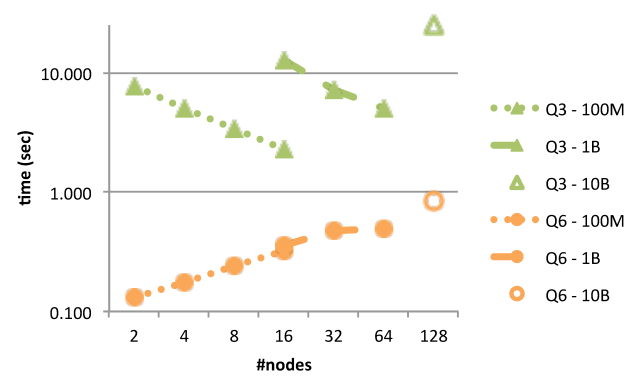

(d) Average query times, over three runs, for the most (Q3) and least (Q6) scalable queries varying datasets and number of nodes

Figure 17: Times to ingest BSBM datasets and execute BSBM queries 1-6

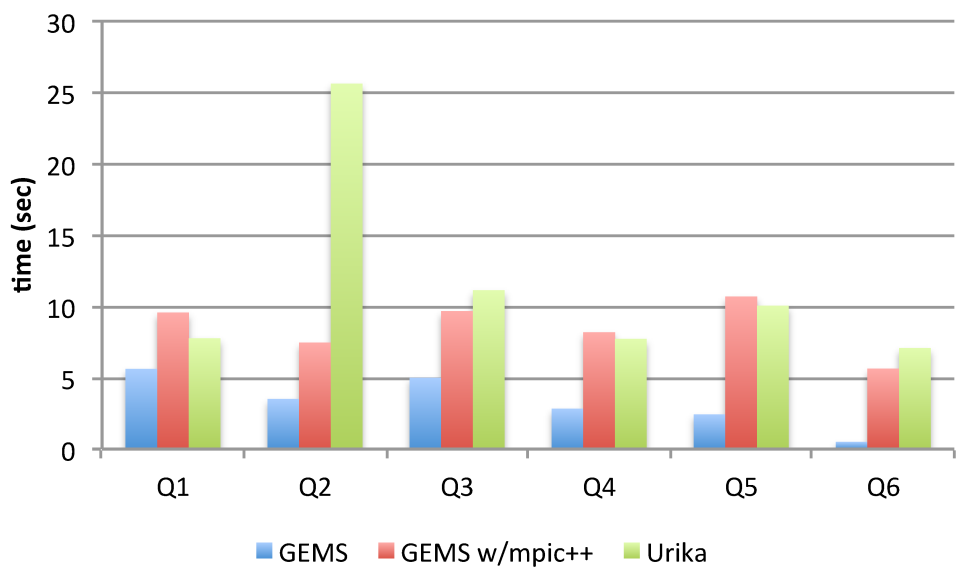

Figure 18: Comparison of GEMS with 64 nodes and Urika, both having 4 TB total memory, with 1B triples; caveats in Section 4.3 


\begin{tabular}{|c|c|c|c|c|c|c|c|c|}
\hline dataset & \multicolumn{9}{|c|}{ 100M } & \multicolumn{3}{|c|}{ 1B } & 10B \\
\hline \#nodes & 2 & 4 & 8 & 16 & 16 & 32 & 64 & 128 \\
\hline ingest & 368 & 185 & 104 & 61.7 & 581 & 345 & 229 & 5085 \\
Q1 & 4.74 & 4.61 & 3.35 & 2.65 & 9.89 & 6.41 & 5.61 & 67.2 \\
Q2 & 1.46 & 1.50 & 1.71 & 1.70 & 2.99 & 3.01 & 3.50 & 5.30 \\
Q3 & 7.67 & 4.93 & 3.44 & 2.26 & 12.8 & 7.21 & 4.99 & 24.6 \\
Q4 & 1.48 & 1.42 & 1.35 & 1.69 & 2.54 & 2.50 & 2.72 & 5.69 \\
Q5 & 0.824 & 0.873 & 1.02 & 1.30 & 1.87 & 1.99 & 2.39 & 4.20 \\
Q6 & 0.130 & 0.172 & 0.238 & 0.316 & 0.358 & 0.482 & 0.484 & 0.835 \\
\hline
\end{tabular}

Table 2: Average time (in seconds) over three runs; to ingest datasets and execute BSBM queries 1-6 with 100M, 1B, and 10B triples; varying number of nodes

\begin{tabular}{|c|r|r|c|}
\hline System & GEMS & GEMS w/mpic++ & Urika \\
\hline ingest & 229 & & 749 \\
Q1 & 5.63 & 9.57 & 7.78 \\
Q2 & 3.52 & 7.46 & 25.6 \\
Q3 & 5.01 & 9.67 & 11.1 \\
Q4 & 2.83 & 8.20 & 7.72 \\
Q5 & 2.44 & 10.7 & 10.0 \\
Q6 & 0.510 & 5.63 & 7.09 \\
\hline
\end{tabular}

Table 3: Comparison of GEMS with 64 nodes and Urika, both having 4 TB total memory, with 1B triples; caveats in Section 4.3

\section{Related Work}

In this section, we briefly cover related work. Cai and Frank [38] presented a RDF query system over a peer-to-peer network. Liarou, Idreos, and Koubarakis [39] implemented RDF query functionality on distributed hash tables. These types of distributed systems differ from ours in that they are more remotely distributed. An interconnected cluster is more tightly connected and is thus "distributed" at a different scale. Works on implementing RDF databases on clusters include YARS2 [18], 4store [16], graph matching using distributed hash joins [22], and so-called evolutionary algorithms [23]. Whereas we implement a distinct, custom, runtime system to support distributed computation, the four aforementioned works interface directly to low-level operations and do not perform the kinds of hardware-aware optimizations performed in GMT. Mapping SPARQL queries to PIG [20, 21] also aims at answering SPARQL queries on clusters, abstracting operations over the PIG language which is compiled into MapReduce jobs. We abstract operations at a lower level, using data structures from SGLIB. There has also been work on RDF databases on shared memory architectures such as the Cray XMT [40]; it is unclear what parts of [40] (if any) are part of Urika. The Cray XMT paradigm is fundamentally different from distributed memory on clusters, as previously discussed in Section 4.4. Commercial, cluster-based 
products for parallel SPARQL queries include OWLIM-Enterprise [19] (replication cluster implemented in Java) and Virtuoso cluster [15] (based on a

distributed column store). There also exist non-RDF oriented graph engines such as GraphX [41].

\section{Conclusions and Future Work}

This paper was motivated by the need to answer analytic queries over science metadata. Science metadata is very heterogeneous, and we postulated that the RDF data model can be used to solve that problem. We illustrated how we used RDF to cope with heterogeneity on the RDESC project in Section 2, also casting questions over the metadata as SPARQL queries. The fundamental operation in SPARQL is graph matching, and thus there is a need for systems that can scale to answer queries over larger graphs. For this reason, we turned to GEMS, and graph engine developed at PNNL. We described GEMS in Section 3 with emphasis on the features that make it suitable for graph walking. In Section 4.2, we demonstrated GEMS' ability to answer RDESC queries over science metadata in the form of 1.4 billion graph edges (RDF triples) on the order of seconds. In Section 4.3, we demonstrated GEMS' ability to scale to larger datasets using the BSBM benchmark. In Section 4.4, we compared the performance of GEMS with Urika.

For RDESC, future work includes enriching the science metadata and continuing to investigate scalability issues in question answering over science metadata. For GEMS, there is ongoing development, particularly maturation of the SPARQL-to-C++ compiler. We are currently pursuing design changes that should enable us to perform queries over 100B triples. We also hope to compare GEMS with other cluster-based solutions like 4store and Virtuoso Cluster Edition.

\section{Acknowledgments}

This material is based in part upon work supported by the U.S. Department of Energy, Office of Science, Office of Advanced Scientific Computing Research, under award number 62892 "Resource Discovery for Extreme Scale Collaboration" (Program Manager Richard Carlson). This material is based in part upon work at the Center for Adaptive Supercomputing Software (CASS) at the U.S. Department of Energy's Pacific Northwest National Laboratory. Pacific Northwest National Laboratory is operated by Battelle 
Memorial Institute under Contract DE-ACO6-76RL01830. A portion of the research was performed using PNNL Institutional Computing.

\section{References}

[1] A. J. Hey, S. Tansley, K. M. Tolle, et al., The fourth paradigm: dataintensive scientific discovery, Microsoft Research Redmond, WA, 2009.

[2] Y. Shafranovich, Common format and MIME type for comma-separated values (CSV) files, RFC4180, IETF, http://tools.ietf.org/html/rfc4180 [last accessed August 14, 2014] (Oct. 2005).

[3] G. Klyne, J. J. Carroll, B. McBride, Resource description framework (RDF): Concepts and abstract syntax, W3C Recommendation, W3C, Cambridge, MA, http://www.w3.org/TR/2004/REC-rdfconcepts-20040210/ [last accessed June 9, 2014] (Feb. 2004).

[4] National Aeronautics and Space Administration, Global change master directory, Online, version 9.9. http://gcmd.nasa.gov/ [last accessed June 9, 2014] (2013).

[5] R. Rew, G. Davis, Netcdf: an interface for scientific data access, Computer Graphics and Applications, IEEE 10 (4) (1990) 76-82.

[6] U.S. Department of Energy Office of Science, ARM climate research facility, Online, http://www.arm.gov/ [last accessed August 14, 2014] (2014).

[7] International Soil Moisture Network, International soil moisture network: ISMN, Online, http://ismn.geo.tuwien.ac.at/ [last accessed June 9, 2014] (2014).

[8] T. Kauppinen, G. M. de Espindola, J. Jones, A. Sánchez, B. Gräler, T. Bartoschek, Linked brazilian amazon rainforest data, Semantic Web.

[9] G. Atemezing, O. Corcho, D. Garijo, J. Mora, M. Poveda-Villalón, P. Rozas, D. Vila-Suero, B. Villazón-Terrazas, Transforming meteorological data into linked data, Semantic Web 4 (3) (2013) 285-290.

[10] J. A. Miñarro-Giménez, M. Egaña Aranguren, B. Villazón-Terrazas, J. T. Fernández Breis, Translational research combining orthologous genes and human diseases with the ogolod dataset, Semantic Web. 
[11] M. Samwald, A. Jentzsch, C. Bouton, C. S. Kallesøe, E. Willighagen, J. Hajagos, M. S. Marshall, E. Prud'hommeaux, O. Hassanzadeh, E. Pichler, et al., Linked open drug data for pharmaceutical research and development, Journal of cheminformatics 3 (1) (2011) 19.

[12] U. Consortium, et al., The universal protein resource (uniprot), Nucleic acids research 36 (suppl 1) (2008) D190-D195.

[13] A. Chappell, S. Choudhury, J. Feo, D. Haglin, A. Morari, S. Purohit, K. Schuchardt, A. Tumeo, J. Weaver, O. Villa, Toward a data scalable solution for facilitating discovery of scientific data resources, in: Proceedings of the 2013 International Workshop on Data-Intensive Scalable Computing Systems, ACM, 2013, pp. 55-60.

[14] P. Boncz, M.-D. Pham, BSBM V3.1 results (April 2013), Online, http://wifo5-03.informatik.unimannheim.de/bizer/berlinsparqlbenchmark/results/V7/index.html [last accessed June 9, 2014] (Apr. 2013).

[15] OpenLink, 6.3. virtuoso cluster operation, Online, http://docs.openlinksw.com/virtuoso/clusteroperation.html [last accessed August 14, 2014].

[16] S. Harris, N. Lamb, N. Shadbolt, The design and implementation of a clustered RDF store, in: Proceedings of the 5th International Workshop on Scalable Semantic Web Knowledge Base Systems, 2009.

[17] Garlik, 5store - 4store, Online, http://4store.org/trac/wiki/5store [last accessed August 14, 2014] (2014).

[18] A. Harth, J. Umbrich, A. Hogan, S. Decker, YARS2: A federated repository for querying graph structured data from the web, in: Proceedings of the 6th International Semantic Web Conference and the 2nd Asian Semantic Web Conference, 2007.

[19] Ontotext, OWLIM editions - ontotext, Online, http://www.ontotext.com/owlim/editions [last accessed August 14, 2014] (2014). 
[20] A. Schätzle, M. Przyjaciel-Zablocki, G. Lausen, PigSPARQL: mapping SPARQL to pig latin, in: Proceedings of the International Workshop on Semantic Web Information Management, 2011.

[21] S. Kotoulas, J. Urbani, P. A. Boncz, P. Mika, Robust runtime optimization and skew-resistant execution of analytical SPARQL queries on pig, in: Proceedings of the 11th International Semantic Web Conference, 2012, pp. 247-262.

[22] J. Weaver, G. T. Williams, Scalable RDF query processing on clusters and supercomputers, in: Proceedings of the 5th International Workshop on Scalable Semantic Web Knowledge Base Systems, 2009.

[23] E. Oren, C. Gueret, S. Schlobach, Anytime query answering in RDF through evolutionary algorithms, in: Proceedings of the 7th International Semantic Web Conference, 2008.

[24] Aurelius, Titan: Distributed graph database, Online, http://thinkaurelius.github.io/titan/ [last accessed August 14, 2014] (2014).

[25] P. Rathle, 2013: What's coming next in Neo4j!, Online, http://neo4j.com/blog/2013-whats-coming-next-in-neo4j/ [last accessed August 14, 2014] (Jan. 2013).

[26] A. Morari, V. G. Castellana, A. Tumeo, J. Weaver, D. Haglin, J. Feo, S. Choudhury, O. Villa, Scaling semantic graph databases in size and performance, Accepted for publication in IEEE Micro, 2014.

[27] J. Weaver, A scalability metric for parallel computations on large, growing datasets (like the web), in: Proceedings of the Joint Workshop on Scalable and High-Performance Semantic Web Systems, 2012.

[28] A. Morari, O. Villa, A. Tumeo, D. G. Chavarria, M. Valero, Scaling irregular applications through data aggregation and software multithreading, in: Proceedings of the 28th IEEE International Parallel and Distributed Processing Symposium, 2014.

[29] A. Morari, V. G. Castellana, D. Haglin, J. Feo, J. Weaver, A. Tumeo, O. Villa, Accelerating semantic graph databases on commodity clusters, 
in: 2013 IEEE International Conference on Big Data, IEEE, 2013, pp. $768-772$.

[30] YarcData, Inc. Urika Big Data Graph Appliance, http://www.cray.com/Products/BigData/uRiKA.aspx [last accessed June 9, 2014].

[31] S. Harris, A. Seaborne, E. Prud'hommeaux, SPARQL 1.1 query language, W3C Recommendation, W3C, Cambridge, MA, http://www.w3.org/TR/2013/REC-sparql11-query-20130321/ [last accessed June 9, 2014] (Mar. 2013).

[32] T. H. Group, The HDF group - information, support, and software, Online, http://www.hdfgroup.org/ [last accessed August 14, 2014] (2014).

[33] GeoNames, GeoNames, Online, http://www.geonames.org/ [last accessed August 14, 2014] (2014).

[34] S. Harris, A. Seaborne, E. Prud'hommeaux, SPARQL 1.1 query language, W3C Working Draft, W3C, Cambridge, MA, http://www.w3.org/TR/2012/WD-sparql11-query-20120105/ [last accessed August 14, 2014] (Jan. 2012).

[35] M. Schmidt, M. Meier, G. Lausen, Foundations of SPARQL query optimization, in: Proceedings of the 13th International Conference on Database Theory, 2010, pp. 4-33.

[36] C. Bizer, A. Schultz, Berlin SPARQL benchmark (BSBM) - business intelligence use case 3.1, Online, http://wifo5-03.informatik.unimannheim.de/bizer/berlinsparqlbenchmark/spec/20110607/

BusinessIntelligenceUseCase/ [last accessed August 14, 2014] (Jul. 2013).

[37] P. Konecny, Introducing the Cray XMT, in: Proc. Cray User Group meeting (CUG 2007), 2007.

[38] M. Cai, M. Frank, RDFPeers: A scalable distributed RDF repository baesd on a structured peer-to-peer network, in: Proceedings of the 13th International World Wide Web Conference, 2004. 
[39] E. Liarou, S. Idreos, M. Koubarakis, Continuous RDF query processing over DHTs, in: Proceedings of the 6th International Semantic Web Conference and the 2nd Asian Semantic Web Conference, 2007.

[40] E. L. Goodman, E. Jimenez, D. Mizell, S. al Saffar, B. Adolf, D. Haglin, High-performance computing applied to semantic databases, in: Proceedings of the 8th Extended Semantic Web Conference, 2011.

[41] R. S. Xin, J. E. Gonzalez, M. J. Franklin, I. Stoica, GraphX: A resilient distributed graph system on spark, in: First International Workshop on Graph Data Management Experiences and Systems, ACM, 2013, p. 2. 